\title{
Anfípodos hiperídeos (Crustacea: Peracarida) del Parque Nacional Isla del Coco, Costa Rica, Pacífico Tropical Oriental
}

\author{
Rebeca Gasca $^{1}$ \& Álvaro Morales-Ramírez ${ }^{2,3}$ \\ 1. El Colegio de la Frontera Sur (ECOSUR) Unidad Chetumal, Av. Centenario Km 5.5 Chetumal 77014, Quintana Roo, \\ México; rgasca@ecosur.mx \\ 2. Centro de Investigación en Ciencias del Mar y Limnología (CIMAR), Ciudad de la Investigación, Universidad de \\ Costa Rica, San Pedro, 11501-2060 San José, Costa Rica; alvaro.morales@ucr.ac.cr \\ 3. Escuela de Biología, Universidad de Costa Rica, San Pedro, 11501-2060 San José, Costa Rica
}

Recibido 02-III-2012. C Corregido 28-VI-2012. Aceptado 24-IX-2012.

\begin{abstract}
Hyperiid amphipods (Crustacea: Peracarida) of the Parque Nacional Isla del Coco, Costa Rica, Eastern Tropical Pacific. Hyperiid amphipods are one of the most frequently encountered pelagic crustaceans and among the most diverse marine zooplankters. To determine the species composition of the hyperiid amphipods dwelling in the waters around Isla del Coco National Park, Pacific Costa Rica, we analyzad 12 zooplankton samples, collected by vertical and horizontals tows with plankton net with mesh sizes of 200 and 500 microns and $49 \mathrm{~cm}$ mouth diameter. We recorded a total of 22 species belonging to 17 genera and 12 families. A faunistic list of this group is provided together with data on their taxonomy and their distribution both in the surveyed area and in general. Most of the species recorded in this zone have been reported in previous surveys in the Eastern Tropical Pacific region and particularly in oceanic waters of Costa Rica. We found eight species representing new records for Costa Rican waters, thus increasing by $26 \%$ (to 38 ) the number of hyperiid species known from Costa Rica. The most frequent species were Lestrigonus shoemakeri, L. bengalensis, Hyperiodes sibaginis, and Phronimopsis spinifera. We expect that additional samplings both from surface and deep waters will expand the knowledge of the diversity of the group in this important protected area. Citation: Gasca, R. \& Á. MoralesRamírez. 2012. Anfípodos hiperídeos (Crustacea: Peracarida) del Parque Nacional Isla del Coco, Costa Rica, Pacífico Tropical Oriental. Rev. Biol. Trop. 60 (Suppl. 3): 223-233. Epub 2012 Dec 01.
\end{abstract}

Key words: amphipods, Hyperiidea, Eastern Tropical Pacific, marine zooplankton.

Los anfípodos hiperídeos son uno de los grupos de crustáceos pelágicos más frecuentes en la comunidad del zooplancton marino; se distribuyen tanto en la zona oceánica como en la nerítica y en un amplio intervalo de profundidad (Vinogradov et al. 1996). Se conocen más de 280 especies anfípodos hiperídeos, por lo que es uno de los grupos más diversos entre los crustáceos pelágicos; para el Océano Pacífico se conocen aproximadamente 222 especies (Vinogradov et al. 1996, Vinogradov 1999). La mayor parte de las especies de hiperídeos se asocian en distintos grados con grupos del zooplancton gelatinoso (Gasca \& Haddock 2004,
Gasca et al. 2009). A pesar de su diversidad y amplia distribución en los grandes océanos, aún existen extensas áreas, sobre todo en las regiones tropicales, en las que sólo se infiere cuáles son las especies que podrían habitarlas, pero en las que no se han hecho muestreos para observar comunidades particulares. Tal es el caso de la fauna planctónica del Pacífico Tropical Oriental y en particular de los alrededores del Parque Nacional Isla del Coco, Costa Rica.

En una de las islas adyacentes a I. Coco, Whitney \& Motta (2008) observaron un enjambre de hiperídeos en interacción con tiburones aunque no pudieron identificar con certeza al 
hiperídeo, ni la naturaleza exacta de la interacción. En un trabajo con datos preliminares acerca del plancton de Isla del Coco, MoralesRamírez (2008) mencionó que se han observado alrededor de 20 especies de hiperídeos con Lestrigonus como el género más común, pero no incluyó una lista de las especies. Adicionalmente, en el trabajo de Gasca (2009a), acerca de los hiperídeos registrados en aguas de Costa Rica, se presentó una lista de 30 especies reconocidas en el Pacífico costarricense, algunas de ellas recolectadas en Bahía Culebra, Golfo de Papagayo. Gasca (2009a) menciona que en el pasado se han presentado problemas en la identificación de las especies de la zona oceánica, por lo que se considera que son necesarios nuevos muestreos para identificar correctamente estos organismos y establecer o corroborar los registros de manera confiable.

La Isla del Coco es una isla oceánica que tiene una superficie de 173000 ha y está situada a $530 \mathrm{~km}$ fuera de la costa Pacífica de Costa Rica. Es un Parque Nacional, así como es un sitio declarado como Patrimonio de la Humanidad; y conforma el núcleo del Área de Conservación Marina Isla del Coco (Cortés 2008). A pesar de su importancia y de la diversidad de ambientes que contiene, no se tienen aún listas completas de especies de la biota marina de la zona. En el caso de los hiperídeos sólo se han publicado datos generales (Morales-Ramírez 2008). El objetivo de este estudio es proporcionar por primera vez, una lista comentada de las especies recolectadas alrededor del Parque Nacional Isla del Coco con datos acerca de su distribución local y regional.

\section{MATERIALES Y MÉTODOS}

El material zooplanctónico analizado proviene de los alrededores de la Isla del Coco $\left(05^{\circ} 31^{\prime} 08^{\prime \prime} \mathrm{N}\right.$ y $\left.87^{\circ} 04^{\prime} 18^{\prime \prime} \mathrm{W}\right)$, la cual se encuentra rodeada por el Océano Pacífico, en la Provincia de la Contracorriente Ecuatorial del Pacífico Norte. Esta zona está influenciada por la Contracorriente Norecuatorial, que fluye débilmente hacia el este a través del océano hacia Centroamérica para girar luego al norte; está además asociada al Domo de Costa Rica (Longhurst 2007, Lizano 2008). En el otro sentido, de este a oeste, recibe influencia de la Corriente Norecuatorial. La zona de elevamiento de la termoclina del Domo de Costa Rica se presenta justo al norte de la zona de estudio y su intensificación estacional (julio a noviembre) muy probablemente tiene importancia ecológica en el área de estudio.

Las muestras de zooplancton analizadas se recolectaron a finales de noviembre de 2001 alrededor de la Isla del Coco en seis estaciones y en el paralelo $8^{\circ} \mathrm{W}$. Las estaciones de muestreo reflejan diferencias ambientales entre el norte y sur de la Isla. Las muestras se obtuvieron mediante arrastres verticales $(0-50 \mathrm{~m})$ y horizontales (3-5min, $1 \mathrm{~m}$ de profundidad), con redes de $0.49 \mathrm{~m}$ de diámetro de boca y apertura de malla de 200 y 500 micras. Se realizaron arrastres tanto de día como de noche y se midió el volumen de agua filtrado con un flujómetro. Las muestras se fijaron en formaldehído al 4\% y fueron preservadas en alcohol al 70\%. Los hiperídeos fueron separados de las muestras disponibles e identificados con la ayuda de las claves y descripciones de los trabajos de Vinogradov et al. (1996), Vinogradov (1999) y Zeidler (2004). No se cuenta con todos los datos para todas las muestras, por lo que se manejarán en resultados sólo los disponibles. Se mencionan los datos de sexo de los ejemplares siempre que fue factible hacerlo. Se incluyen fotografías de calidad aceptable obtenidas con una cámara Nikon E5400 y un microscopio de disección Nikon SMZ 1000. Las muestras originales y los especímenes identificados se encuentran depositados en el Museo de Zoología de la Universidad de Costa Rica.

\section{RESULTADOS}

Se registraron 22 especies de anfípodos hiperídeos, todas pertenecientes al Infraorden Physocephalata. Se presenta a continuación una lista comentada de las especies encontradas en el zooplancton de las aguas que rodean la Isla del Coco. Se sigue en general el arreglo taxonómico propuesto por Vinogradov (1999) 
y en grupos particulares se siguen los criterios de Zeidler (2003, 2004).

\section{Orden AMPHIPODA}

Suborden HYPERIIDEA Milne-Edwards, 1830 Infraorden PHYSOCEPHALATA Bowman \& Gruner, 1973

Superfamilia VIBILIOIDEA Bowman \& Gruner, 1973

Familia VIBILIIDAE Dana, 1852

Vibilia chuni Behning \& Woltereck, 1912

Material examinado: un ejemplar de Bahía Yglesias, en un arrastre vertical, diurno (Fig. 1A).

Es una especie rara que se encuentra en las aguas tropicales del mundo y de manera abundante en la costa este del sur de África (Zeidler 2003). Esta especie no había sido registrada en aguas de Costa Rica.

\section{Familia PARAPHRONIMIDAE Bovallius,} 1887

\section{Paraphronima gracilis Claus, 1879}

Material examinado: un ejemplar de Bahía Chatham (Fig. 1B).

Especie de aguas cálidas, circumtropical y distribuida en los primeros $500 \mathrm{~m}$ de profundidad (Vinogradov 1999); se ha encontrado abundante y ampliamente distribuida en la Corriente de California (Bowman 1973). Ha sido registrada en aguas del Domo de Costa Rica (Salmán-Palacios 1985).

Superfamilia PHRONIMOIDEA Bowman \& Gruner, 1973

Familia PHRONIMIDAE Dana, 1852

\section{Phronima bowmani Shih, 1991}

Material examinado: cuatro ejemplares recolectados en arrastres horizontales y verticales en las bahías Chatham y Wafer (diurno) (Fig. 1C).
Especie del Pacífico Tropical Oriental, muy similar a $P$. colleti y a $P$. bucephala (Shih 1991). La validez de esta especie fue cuestionada por Vinogradov et al. (1996), quienes piensan que es indiferenciable de $P$. bucephala, pero los especímenes encontrados se apegan más a la descripción de $P$. bowmani y además se encuentra en la misma región de estudio. Este representa el primer registro de esta especie en aguas de Costa Rica.

Familia PHROSINIDAE Dana, 1852

\section{Phrosina semilunata Risso, 1822}

Material examinado: un ejemplar recolectado en un arrastre vertical en Bahía Yglesias, en un arrastre vertical, diurno (Fig. 1D).

Es muy común y abundante en aguas tropicales, especialmente en las aguas cercanas a la costa y está entre las especies de hiperídeos más abundantes en el Pacífico oriental (Shih $\&$ Chen 1995). Se ha registrado en aguas del Domo de Costa Rica (Salmán-Palacios 1985, Gasca 2009a).

\section{Primno brevidens Bowman, 1978}

Material examinado: un ejemplar recolectado en un arrastre vertical en Bahía Yglesias, en un arrastre vertical, diurno.

Es una de varias especies del género con talla intermedia $(9 \mathrm{~mm})$. Se puede diferenciar de congéneres similares (al igual que $P$. evansi Sheader, 1986) por el corto proceso dentiforme en el margen anterior del carpo del pereópodo 5. De hecho, puede confundirse con $P$. evansi pero según Zeidler (2004) está limitada a aguas tropicales del Pacífico y $P$. evansi al Atlántico Norte. Existen trabajos que la determinan como una de las especies más abundantes de la Corriente de California, principalmente en aguas cálidas y cercanas a la costa (Bowman 1978) y en la zona de transición al sur de la Corriente de California y el Golfo de California (Gasca et al. 2010). Tal como sucede en otras especies del género, los registros regionales y patrones de distribución de $P$. brevidens deben 

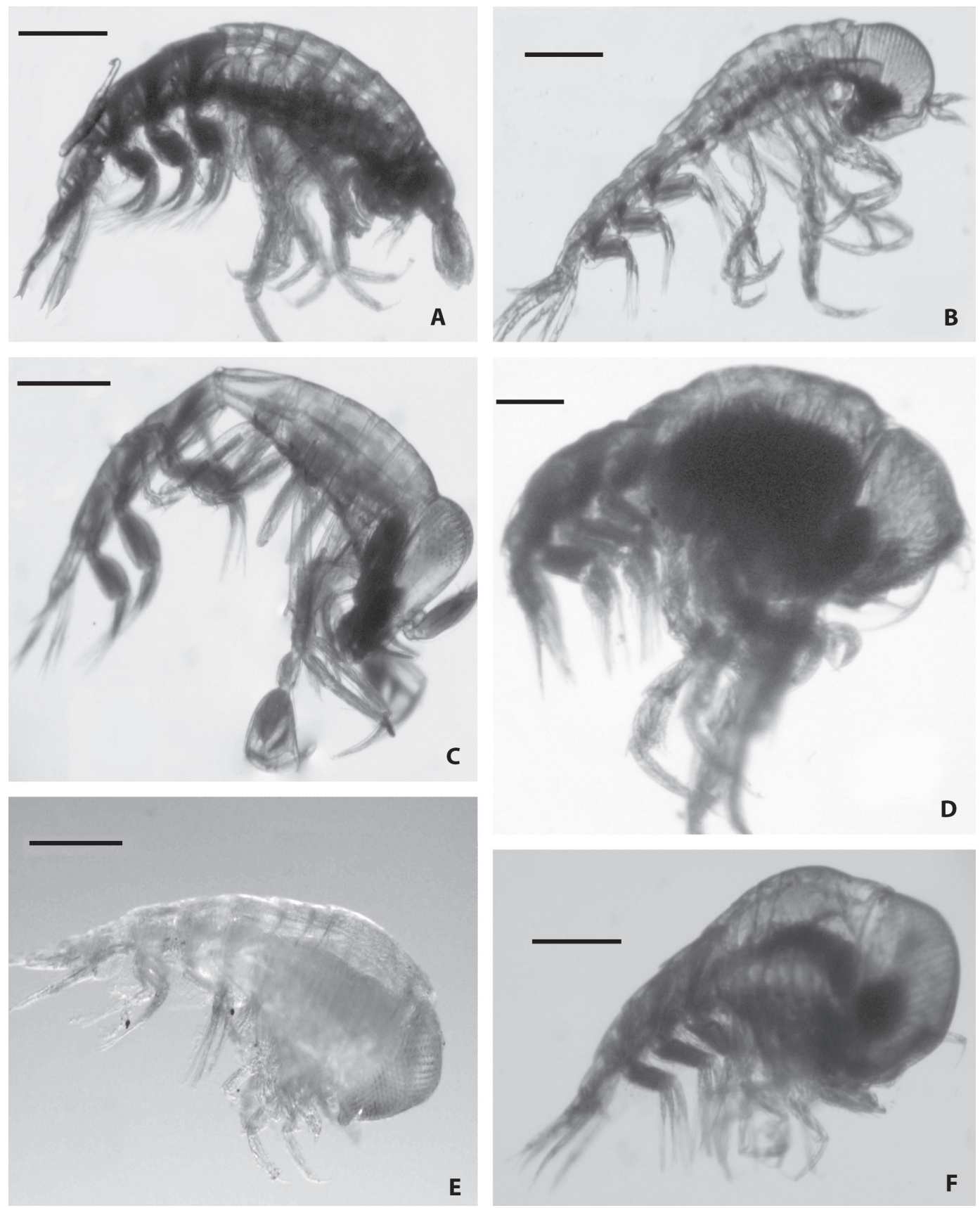

Fig. 1. Especies de anfípodos hiperídeos recolectadas en el Parque Nacional Isla del Coco, Costa Rica. (A) Vibilia chuni. (B) Paraphronima gracilis. (C) Phronima bowmani. (D) Phrosina semilunata. (E) Lestrigonus bengalensis. (F) Lestrigonus macrophthalmus. Escala $=1 \mathrm{~mm}(\mathrm{~A}-\mathrm{D}) ;=0.5 \mathrm{~mm}(\mathrm{E}, \mathrm{F})$.

Fig. 1. Species of hyperiid amphipods collected at the Parque Nacional Isla del Coco, Costa Rica. (A) Vibilia chuni. (B) Paraphronima gracilis. (C) Phronima bowmani. (D) Phrosina semilunata. (E) Lestrigonus bengalensis. (F) Lestrigonus macrophthalmus. Scale bars $=1 \mathrm{~mm}(\mathrm{~A}-\mathrm{D}) ;=0.5 \mathrm{~mm}(\mathrm{E}, \mathrm{F})$. 
ser revisados. Se ha registrado en aguas del Domo de Costa Rica (Salmán-Palacios 1985, Gasca 2009b).

Familia LESTRIGONIDAE Zeidler, 2004

\section{Lestrigonus bengalensis Giles, 1887}

Material examinado: nueve ejemplares recolectados tanto en Bahía Chatham como en Bahía Wafer tanto en arrastres diurnos como nocturnos y en una estación oceánica ubicada en el paralelo $8^{\circ} \mathrm{W}$ (Fig. 1E).

Muy común en aguas tropicales, especialmente en las zonas cercanas a la costa (Gasca et al. 2012). Encontrada como una de las más abundantes en aguas tropicales del Pacífico oriental y el Mar Caribe (Gasca 2009 a,b, Gasca et al. 2012). Ha sido encontrada en aguas de Costa Rica (Gasca 2009b).

\section{Lestrigonus macrophthalmus}

(Vosseler, 1901)

Material examinado: una hembra en Bahía Chatham y un macho en la estación externa de Bahía Wafer (Fig. 1F).

Es común en las zonas tropical y templada de los océanos (Zeidler 1998) y no había sido previamente registrada en aguas de Costa Rica.

\section{Lestrigonus schizogeneios}

(Stebbing, 1888)

Material examinado: se recolectaron más de 20 ejemplares, tanto machos como hembras en las bahías Chatham, Wafer e Yglesias (en arrastre diurno, vertical) (Fig. 2A).

Especie circumtropical que habita los primeros $200 \mathrm{~m}$ de profundidad (Vinogradov 1999); esta especie ha sido observada en Bahía Culebra, Golfo de Papagayo, Costa Rica (Gasca 2009b).

Lestrigonus latissimus (Bovallius, 1889)

Material examinado: una hembra en estación externa de Bahía Wafer (Fig. 2B).
Especie de aguas epipelágicas tropicales (Vinogradov 1999) que ha sido encontrada previamente en aguas costeras del Pacífico costarricense (Gasca 2009b).

\section{Lestrigonus shoemakeri Bowman, 1973}

Material examinado: $>30$ machos, $>20$ hembras (Fig. 2C). Fue la especie con mayor abundancia en las muestras de Isla del Coco; se encontraron ejemplares de ambos sexos tanto en Bahía Chatham como en Bahía Wafer, en arrastres verticales y horizontales, y diurnos y nocturnos.

Es una especie común en el Pacífico ecuatorial oriental (Bowman 1978), encontrada como la tercera más abundante en aguas del Golfo de California (Siegel-Causey 1980). Fue registrada previamente en Bahía Culebra, Golfo de Papagayo, Pacífico de Costa Rica (Gasca 2009b).

\section{Phronimopsis spinifera Claus, 1879}

Material examinado: nueve ejemplares en Bahía Chatham, en el paralelo $8^{\circ} \mathrm{W}$ y con mayor frecuencia en Bahía Yglesias en una estación diurna vertical (Fig. 2D).

Especie circumtropical, no muy común pero fácilmente reconocible por el tamaño y estructura de su quela. Ha sido encontrada hasta los $500 \mathrm{~m}$ de profundidad (Vinogradov 1999); fue observada por Gasca (2009b) en el Pacífico de Costa Rica.

\section{Hyperioides sibaginis (Stebbing, 1888)}

Material examinado: se recolectaron nueve ejemplares en las bahías Chatham, Wafer e Yglesias, siempre uno o dos ejemplares tanto en arrastres verticales como horizontales (Fig. 2E).

Es una de las tres especies más abundantes en el giro central del Pacífico (Shulenberger 1977), la más abundante en aguas de la Gran Barrera de Arrecifes (Barnard 1931) y también en el Pacífico central mexicano (Gasca et al. 2012). En aguas costarricenses del Pacífico 

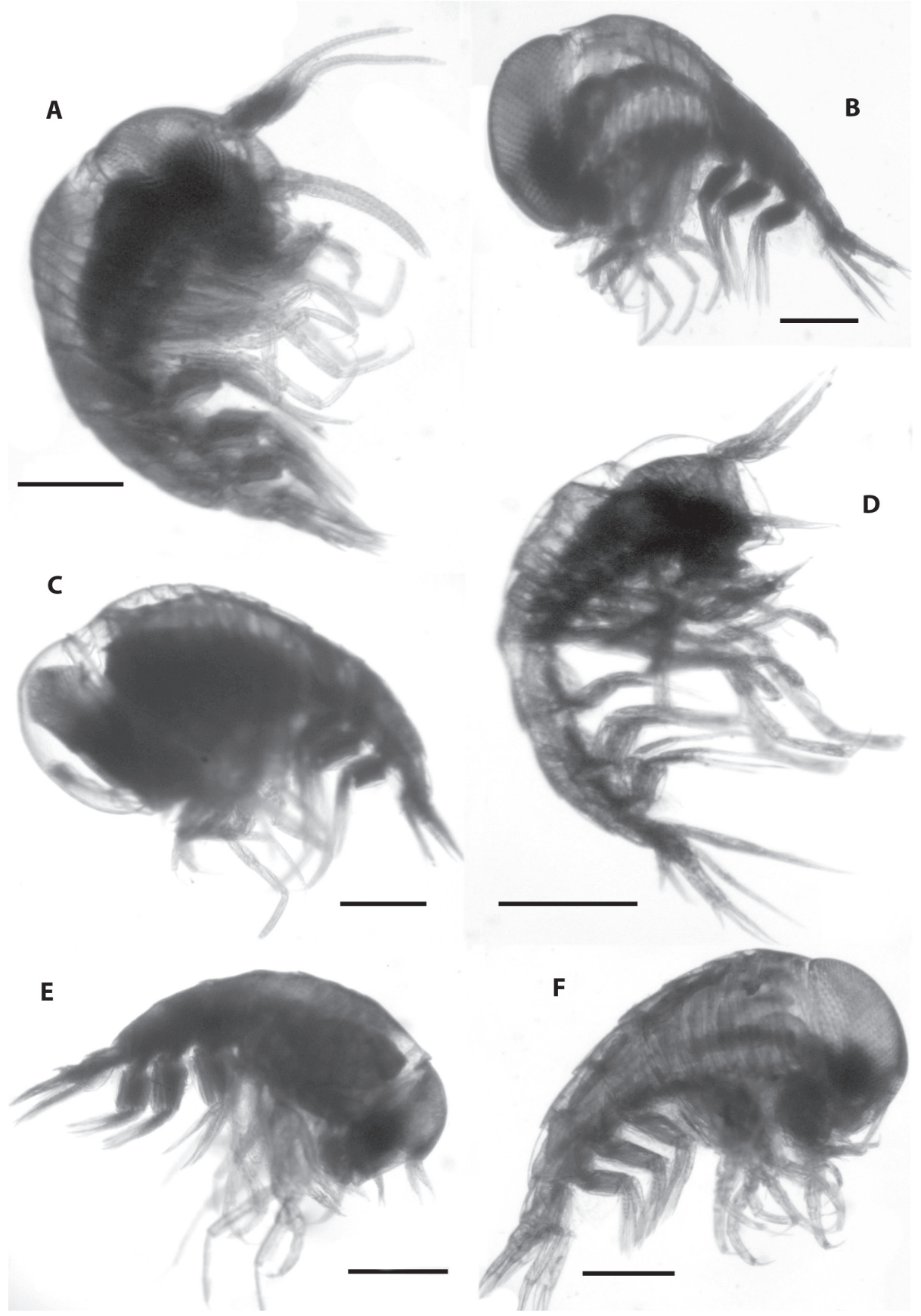

Fig. 2. Especies de anfípodos hiperídeos recolectadas en el Parque Nacional Isla del Coco, Costa Rica. (A) Lestrigonus schizogeneios. (B) Lestrigonus latissimus. (C) Lestrigonus shoemakeri. (D) Phronimopsis spinifera. (E) Hyperioides sibaginis. (F) Hyperietta vosseleri. Escala $=0.5 \mathrm{~mm}(\mathrm{~A}-\mathrm{C}, \mathrm{F}) ;=1 \mathrm{~mm}(\mathrm{D}, \mathrm{E})$.

Fig. 2. Species of hyperiid amphipods collected at the Parque Nacional Isla del Coco, Costa Rica. (A) Lestrigonus schizogeneios. (B) Lestrigonus latissimus. (C) Lestrigonus shoemakeri. (D) Phronimopsis spinifera. (E) Hyperioides sibaginis. (F) Hyperietta vosseleri. Scale bars $=0.5 \mathrm{~mm}(\mathrm{~A}-\mathrm{C}, \mathrm{F}) ; 1.0 \mathrm{~mm}(\mathrm{D}, \mathrm{E})$. 
fue registrada previamente en Bahía Culebra, Golfo de Papagayo (Gasca 2009b).

\section{Hyperietta vosseleri (Stebbing, 1904)}

Material examinado: se obtuvieron tres ejemplares en Chatham en un arrastre vertical y uno horizontal (Fig. 2F).

Es una especie común en la zona epipelágica de aguas tropicales y templado-cálidas de los océanos (Zeidler 1998); fue la segunda más abundante en el giro central del Pacífico (Shulenberger 1977). Fue registrada en Bahía Culebra, Golfo de Papagayo, Costa Rica (Gasca 2009b).

Superfamilia LYCAEPSOIDEA Bowman \& Gruner, 1973

Familia LYCAEPSOIDAE Chevreux, 1913

\section{Lycaeopsis themistoides Claus, 1879}

Material examinado: dos ejemplares recolectados, uno en Bahía Chatham (vertical) y uno en Bahía Wafer (horizontal).

Junto con su congénere L. zamboangae, son especies poco frecuentes pero ampliamente distribuidas en las zonas tropicales y templadas de los océanos, incluyendo el Pacífico Tropical (Zeidler 1998, Gasca \& Franco-Gordo 2008). Este es el primer registro de la especie en aguas de Costa Rica.

\section{Lycaeopsis zamboangae (Stebbing, 1888)}

Material examinado: Un ejemplar de un arrastre horizontal, diurno de la zona de Wafer (Fig. 3A).

Al igual que su congénere L. themistoides, no suele ser muy común, pero sí está ampliamente distribuida en latitudes templadas y cálidas (Dick 1970), pero al parecer sólo es en aguas cálidas que se encuentra por arriba de la termoclina (Thurston 1976). No había sido registrada en aguas de Costa Rica.

Superfamilia PLATYSCELOIDEA Bate, 1862 Familia PRONOIDAE Claus, 1879
Eupronoe armata Claus, 1879

Material examinado: dos machos y dos hembras recolectados en las zonas de bahías Chatham (arrastre vertical) y Wafer (arrastre horizontal) (Fig. 3B).

Especie de aguas cálidas de los océanos. Vinogradov et al. (1996) señalan que es "aparentemente" circumtropical y la sinonimizan con E. intermedia, que se distribuye en el Océano Atlántico, en los mares de China, en el Océano Índico y al este y oeste de Sudáfrica (Shih \& Cheng 1995). Este es el primer registro de la especie para aguas de Costa Rica.

Familia LYCAEIDAE Claus, 1879

\section{Simorhynchotus antennarius (Claus, 1871)}

Material examinado: 2 machos recolectados en Bahía Wafer (arrastre horizontal, diurno) (Fig. 3C).

Una especie circumtropical abundante y ampliamente distribuida hasta los $500 \mathrm{~m}$ de profundidad (Vinogradov 1999). Fue registrada en aguas de Bahía Culebra, Golfo de Papagayo, Costa Rica (Gasca 2009b).

Familia BRACHYSCELIDAE Stephensen, 1923

Brachyscelus crusculum Bate, 1861

Material examinado: Sólo ejemplares juveniles encontrados en Chatham y Wafer (Fig. 3D).

Es una especie común en las zonas tropicales y templadas y de 0 a $300-400 \mathrm{~m}$ de profundidad (Zeidler \& de Broyer 2009). Este es el primer registro de la especie en aguas costarricenses.

\section{Familia OXYCEPHALIDAE}

Oxycephalus clausi Bovallius, 1887

Material examinado: un ejemplar en estaciones exteriores de las bahías Chatham y Wafer (Fig. 3E). 

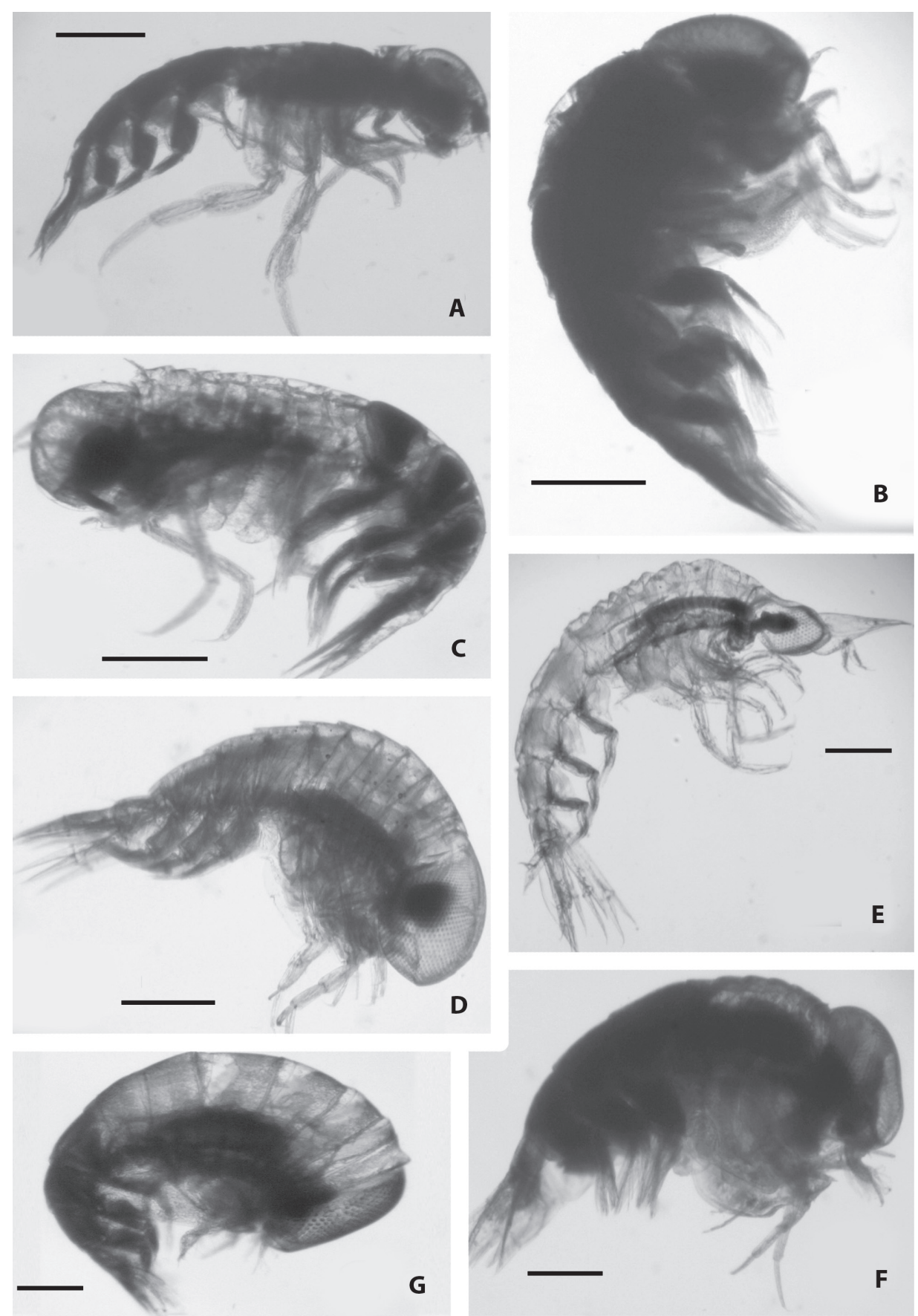

Fig. 3. Especies de anfípodos hiperídeos recolectadas en el Parque Nacional Isla del Coco, Costa Rica. (A) Lycaeopsis zamboangae. (B) Eupronoe armata. (C) Simorhynchotus antennarius. (D) Brachyscelus crusculum. (E) Oxycephalus clausi. (F) Amphithyrus sculpturatus. (G) Parascelus edwardsi. Escala $=1 \mathrm{~mm}(\mathrm{~A}-\mathrm{E}, \mathrm{G}) ;=0.5 \mathrm{~mm}$ (F).

Fig. 3. Species of hyperiid amphipods collected at the Parque Nacional Isla del Coco, Costa Rica. (A) Lycaeopsis zamboangae. (B) Eupronoe armata. (C) Simorhynchotus antennarius. (D) Brachyscelus crusculum. (E) Oxycephalus clausi. (F) Amphithyrus sculpturatus. (G) Parascelus edwardsi. Scale bars $=1 \mathrm{~mm}$ (A-E, G); $=0.5 \mathrm{~mm}$ (F). 
Habita aguas epipelágicas tropicales de los océanos del mundo (Vinogradov 1999); fue registrada previamente por Gasca (2009b) en aguas de Bahía Culebra, Golfo de Papagayo.

\section{Familia PLATYSCELIDAE Bate, 1862}

\section{Tetrathyrus forcipatus Claus, 1879}

Material examinado: 2 machos recolectados en las estaciones exteriores (una nocturna) de Bahía Wafer.

Es común y abundante en aguas superficiales de la zona tropical y templada de los océanos (Zeidler \& de Broyer 2009) y en aguas superficiales, raramente por debajo de los 40 $\mathrm{m}$ de profundidad (Thurston 1976). Este es el primer registro de la especie en aguas de Costa Rica.

\section{Amphithyrus sculpturatus Claus, 1879}

Material examinado: Dos ejemplares recolectados en arrastre horizontal en la estación interior de Bahía Chatham (Fig. 3F).

Especie rara pero ampliamente distribuida que habita los primeros $100 \mathrm{~m}$ de profundidad de las regiones tropicales oceánicas (Vinogradov 1999); fue registrada en aguas de la Bahía Culebra, Golfo de Papagayo en el Pacífico costarricense (Gasca 2009b).

\section{Familia PARASCELIDAE Claus, 1879}

\section{Parascelus edwardsi Claus, 1879}

Material examinado: Dos ejemplares recolectados en la zona externa de Bahía Wafer (una nocturna) (Fig. 3G).

Especie ampliamente distribuida pero poco abundante en las regiones tropicales y templadas de los océanos (Dick 1970), en el Golfo de California fue muy abundante (Siegel-Causey 1982) así como en la parte central del Pacífico mexicano (Gasca et al. 2012). Se ha observado en aguas costeras del Pacífico de Costa Rica (Gasca 2009a).

\section{DISCUSIÓN}

Las especies de hiperídeos encontradas en la zona aledaña al Parque Nacional Isla del Coco tienen, en general, una distribución tropical o circumtropical y pertenecen al Infraorden Physocephalata, grupo del que las especies se distribuyen principalmente en la zona epipelágica. El Infraorden Physosomata podría estar representado en el área de estudio, pero estas son formas de aguas más profundas que las que fueron muestreadas; igualmente, otros Physocephalata son habitantes de aguas mesopelágicas. Es por ello que para tener una lista más completa de las especies de la zona es necesario hacer más muestreos tanto en áreas costeras como oceánicas y a mayor profundidad. En aguas del Caribe occidental muestreos mesopelágicos de zooplancton produjeron un incremento del $30 \%$ en los listados locales (Gasca 2009c).

Con el esfuerzo de muestreo desarrollado en las aguas circundantes de la Isla del Coco se registraron ocho especies de hipéridos que no habían sido previamente encontradas en aguas costarricenses, lo que representa un incremento de $26 \%$ respecto a lo recientemente registrado por Gasca (2009a). La lista podría ser considerablemente más extensa al aumentar la frecuencia de los muestreos y el número de localidades.

Una estación en la zona interna de Bahía Chatam y en un arrastre horizontal (no hay dato de la hora) se encontraron más especies (10).

Las especies encontradas con frecuencia en el área de estudio fueron Lestrigonus shoemakeri, L. schizogeneios, Phronimopsis spinifera, Hyperioides sibaginis y Lestrigonus bengalensis, aunque sin datos de volumen filtrado esto es sólo una apreciación. Hyperioides sibaginis ha sido encontrada como una especie muy abundante en otras zonas del Pacífico (Gasca et al. 2008, 2012), y aquí se encontró frecuentemente pero sólo unos cuantos ejemplares cada vez. De las 22 especies registradas aquí, 16 fueron registradas por Valencia y Giraldo $(2009,2011)$ y 21 por Valencia (2011), encontrando siempre a $H$. sibaginis y $L$. 
bengalensis como las especies más abundantes en aguas del Pacífico tropical oriental en la zona de Colombia. De manera comparativa, en otra zona del Pacífico Oriental, frente a las costas mexicanas, las especies más abundantes fueron $H$. sibaginis, $(65.1 \%), L$. bengalensis (14.2\%), L. schizogeneios (5.5\%), T. forcipatus (1.6\%), L. shoemakeri (1.3\%), P. edwardsi $(1.2 \%)$ y L. zamboangae (1.0\%) (Gasca et al. 2012). Todas esas especies se comparten en las dos zonas (costas del Pacífico central e Isla del Coco), aunque probablemente no en el mismo orden, ni en la misma proporción, pero harán falta más estudios para poder observar claramente las diferencias en la estructura de la comunidad en función de las distintas características ambientales.

Otra particularidad de los hiperídeos es que muchas de las especies, si no es que todas ellas, utilizan a alguna clase de organismos gelatinosos, como las medusas, los sifonóforos o los ctenóforos, como sustrato y alimento por lo menos en etapas tempranas de su vida. Por ello, el conocimiento de la composición, distribución y abundancia de estos organismos gelatinosos también ayudaría a comprender la dinámica de las poblaciones de hiperídeos (Gasca et al. 2009). Los estudios cuantitativos realizados en distintas épocas del año, con condiciones climáticas diferenciadas y tal vez asociadas a la dinámica del Domo de Costa Rica, son también necesarios en la zona de estudio.

\section{AGRADECIMIENTOS}

Agradecemos a Jairo García y Jorge Picado su trabajo en el campo, a Roliér Lara su trabajo en el laboratorio, así como al personal de apoyo del Parque Nacional Isla del Coco. Agradecemos el apoyo de la Vicerrectoría de Investigación de la Universidad de Costa Rica a través de los proyectos 808-3A-062 y 808-B0-060.

\section{RESUMEN}

Los anfípodos hiperídeos son uno de los grupos de crustáceos pelágicos más frecuentemente encontrados y están entre los más diversos del zooplancton marino. Para determinar la composición de especies de anfípodos hipéridos que habitan las aguas alrededor del Parque Nacional Isla del Coco, Costa Rica, se analizaron 12 muestras de zooplancton. Estas fueron recolectadas mediante arrastres verticales y horizontales con una red de plancton con mallas de 200 y 500 micras y $49 \mathrm{~cm}$ de diámetro en la boca. Se encontró un total de 22 especies pertenecientes a 17 géneros y 12 familias. Se presenta el listado faunístico de este grupo junto con datos acerca de su taxonomía, su distribución en el área de estudio y su distribución general. La mayor parte de las especies registradas en esta zona han sido reportadas en estudios previos referentes a la región del Pacífico Tropical Oriental y en particular en aguas oceánicas de Costa Rica. Encontramos ocho especies que representan nuevos registros para aguas del Pacífico costarricense, lo que incrementa en un 26\% (a 38) el número de especies de hipéridos conocidos en Costa Rica. Las especies más frecuentes fueron Lestrigonus shoemakeri, L. bengalensis, Hyperioides sibaginis y Phronimosis spinifera. Se espera que muestreos adicionales tanto de aguas superficiales como profundas expandan el conocimiento de la diversidad del grupo en esta área protegida.

Palabras clave: anfípodos, Hyperiidea, Pacífico tropical oriental, zooplancton marino.

\section{REFERENCIAS}

Barnard, K.H. 1931. Amphipoda. Great Barrier Reef Expedition 1928-29. British Mus. (Nat. Hist.) Sci. Rep. 4: 111-135.

Bowman, T.E. 1973. Pelagic amphipods of the genus Hyperia and closely related genera (Hyperiidea: Hyperiidae). Smithsonian Contributions to Zoology 136: 1-76.

Bowman, T.E. 1978. Revision of the pelagic amphipod genus Primno (Hyperiidea: Phrosinidae). Smithson. Contrib. Zool. 275: 1-23.

Cortés, J. (Ed.). 2008. Investigaciones marinas en la Isla del Coco, Costa Rica. Rev. Biol. Trop. 56 (Supl. 2): $217 \mathrm{p}$.

Dick, R.I. 1970. Hyperiidea (Crustacea: Amphipoda). Key to South African genera and species, and a distribution list. Ann. South Afr. Mus. 57: 25-86.

Gasca, R. 2009a. Hyperiid Amphipods, p. 275-282. In I.S Wehrtman \& J. Cortés (eds). Marine Biodiversity of Costa Rica, Central America. Springer, Berlín.

Gasca, R. 2009b. Hyperiid amphipods (Crustacea: Peracarida) in Mexican waters of the Pacific Ocean. Pac. Sci. 63: 8395 .

Gasca, R. 2009c. Diversity of Siphonophora (Cnidaria: Hydrozoa) in the Western Caribbean Sea: new records from deep-water trawls. Zootaxa 2095: 60-68. 
Gasca, R. \& C. Franco-Gordo. 2008. Hyperiid amphipods (Peracarida) from Banderas Bay, Mexican Tropical Pacific. Crustaceana 81: 115-125.

Gasca, R. \& S.H. Haddock, 2004. Associations between gelatinous zooplankton and hyperiid amphipods (Crustacea: Peracarida) in the Gulf of California. Hydrobiologia 530/531: 529-535.

Gasca, R., H. Manzanilla \& E. Suárez-Morales. 2009. Distribution of hyperiid amphipods (Crustacea) of the Southern Gulf of Mexico, summer and winter 1991. J. Plankton Res. 31: 1493-1504.

Gasca, R., E. Suárez-Morales \& C. Franco-Gordo. 2010. New records of hyperiids (Amphipoda, Hyperiidea) from surface waters of the central Mexican Pacific. Crustaceana 83: 927-940.

Gasca, R., C. Franco-Gordo, E. Godínez-Domínguez \& E. Suárez-Morales. 2012. Hyperiid amphipods community in the Eastern Tropical Pacific before, during, and after El Niño 1997-1998. Mar. Ecol. Progr. Ser.: en prensa.

Lizano, O. 2008. Dinámica de aguas alrededor de la Isla del Coco, Costa Rica. Rev. Biol. Trop. 56 (Suppl. 2): 31-48.

Longhurst, A.R. 2007. Ecological Geography of the Sea. 2a. ed. Elsevier, Amsterdam.

Morales-Ramírez, A. 2008. Caracterización cualitativa del zooplancton del Área de conservación marina Isla del Coco (ACMIC), Océano Pacífico de Costa Rica. Rev. Biol. Trop. 56 (Supl. 2): 159-169.

Salmán-Palacios, C. 1985. Sistemática y distribución de los hiperídeos pelágicos (Crustacea: Amphipoda) del Domo de Costa Rica. Tesis Profesional, Facultad de Ciencias, UNAM, México, D.F.

Shih, C.-t. 1991. Description of two new species of Phronima Latreille, 1802 (Amphipoda: Hyperiidea) with a key to all species of the genus. J. Crust. Biol. 11: $322-335$

Shih, C.-t. \& Q.-c. Chen. 1995. Zooplankton of China Seas (2). The Hyperiidea (Crustacea: Amphipoda): 1-295. China Ocean Press, Beijing, China.

Shulenberger, E. 1977. Hyperiid amphipods from the zooplankton community of the North Pacific central gyre. Mar. Biol. 42: 375-385.
Siegel-Causey, D. 1982. Factors determining the distribution of hyperiid Amphipoda in the Gulf of California. Thesis Ph.D., University of Arizona, Texas, EUA.

Thurston, M.H. 1976. The vertical distribution and diurnal migration of the Crustacea Amphipoda collected during the SOND Cruise, 1965. II. The Hyperiidea and general discussion. J. Mar. Biol. Assoc. UK 56: 383-470.

Valencia, B. y A. Giraldo. 2009. Hipéridos (Crustácea : Amphipoda) en el sector norte del Pacífico oriental tropical colombiano. Lar. Am. J. Aquat. Res., 37(2):265-273.

Valencia, B. y A. Giraldo. 2011. Structure of hyperiid amphipod assemblages on Isla Gorgona, eastern tropical Pacific off Colombia. J. Mar. Biol. Assoc. U.K.: 11doi:10.1017/S0025315411001780

Vinogradov, G.M. 1999. Amphipoda, p. 1141-1240 In D. Boltovskoy (ed). South Atlantic Zooplankton. Backhuys Publishers, Leiden, Holanda.

Vinogradov, M.E., A.F. Volkov \& T.N. Semenova. 1996. Hyperiid Amphipods (Amphipoda, Hyperiidea) of the World Oceans. Science Publ. Inc., Lebanon, Kansas, EUA.

Whitney, N.M. \& P.J. Motta. 2008. Cleaner host posing behavior of whitetip reef sharks (Triaenodon obesus) in a swarm of hyperiid amphipods. Coral Reefs 27:363.

Zeidler, W. 1998. Pelagic amphipods (Crustacea: Amphipoda: Hyperiidea) collected from eastern and southeastern Australian waters by the CSIRO research vessel "Warreen" during the years 1938-1941. Rec. South Austr. Mus., Monogr. Ser. 4: 1-143.

Zeidler, W. 2003. A review of the hyperiidean amphipod superfamily Vibilioidea Bowman and Gruner, 1973 (Crustacea: Amphipoda: Hyperiidea). Zootaxa 280: $1-104$.

Zeidler, W. 2004. A review of the families and genera of the hyperiidean amphipod superfamily Phronimoidea Bowman and Gruner, 1973 (Crustacea: Amphipoda: Hyperiidea). Zootaxa 567: 1-66.

Zeidler, W. \& De Broyer, C. 2009. Catalogue of the Hyperidean Amphipoda (Crustacea) of the Southern Ocean with distribution and ecological data. In C. De Broyer (ed.). Census of Antarctic Marine Life, Synopsis of the Amphipoda of the Southern Ocean. Bull. Inst. Royal Sci. Nat. Belg. 79 (Suppl. 1): 1-103. 
